\title{
Site Automation - Does it pay off?
}

\author{
Ing. Achiel Sturm \\ Topcon Europe Positioning B.V., Capelle a/d IJssel, The Netherlands \\ Achiel.Sturm@Topcon.eu
}

Processes on a job site resemble an industrial manufacturing process. Different stages depend on each other, but are often carried out independently. Automating a job site by utilizing machine control is a way to increase productivity and reduce the operating costs. To meet the tight time schedule for a project one needs to take care of data management, stake-out and execution. This is where machine control (MC) needs to be factored in to allow its implementation into logistics and processes. If MC systems are to be used this has to be planned, prepared and executed by the persons in charge of the site management. Only if the design data is strictly adhered to each additional planning and design stage, machine control can be executed successfully. An efficient process control based on design data is directly turned into an increase in productivity and dramatically cost savings. Critical factors in job site automation are the efficient implementation of design models and especially the task of integrating survey and stake-out jobs into the on-site processes. This needs a direct link between survey results and machine control as well as continuous exchange of data during the full process.

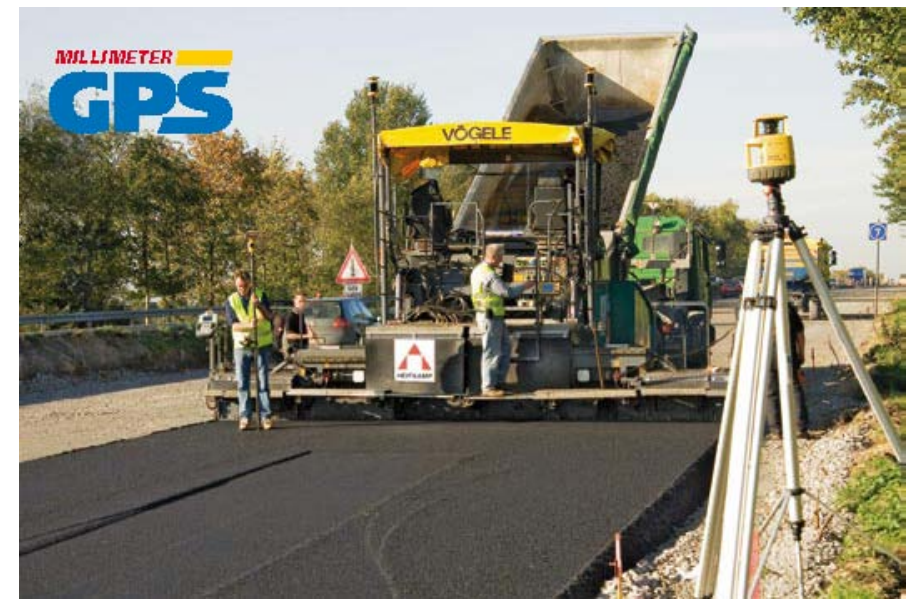

Figure 1: Machine Control and Survey in progress

\section{From elevation checks to three-dimensional automation}

More than 30 years ago, lasers showed up in the construction industry. The perfectly straight laser beam (given a homogeneous medium of constant temperature) was used to simplify pipe laying work. It was not so much the horizontal alignment, but more the laying of pipes with a clearly defined and constant slope. For drainage systems, water accumulations were a persistent problem, thus voiding the effect the drainage was supposed to yield. Using a laser for alignment increased the quality enormously.

\section{Manual control using display systems}

Controlling construction equipment with lasers-a well-known technology on almost any site and in many applications - started with one simple laser beam. The beam plane was and is used as a reference and control plane. 
However, improvement was possible by starting a phase earlier: Why not use a rotating laser while grading the surface itself? For this task, much larger laser receivers, called machine receivers, are used. The simplest application attaches a machine receiver to the blade of a machine, for example a motor grader. Icons or LEDs guide the motor grader operator while he spreads the material: "Blade too high", "Blade too low" or "Blade on grade". This creates a very simple machine control system, called a indicate system. One can build upon this by adding a second receiver to determine the blade slope or by using remote displays that offer a more convenient way for the operator to see the blades level. The capital investment for a simple display system is low, making it an attractive solution even for small construction sites.

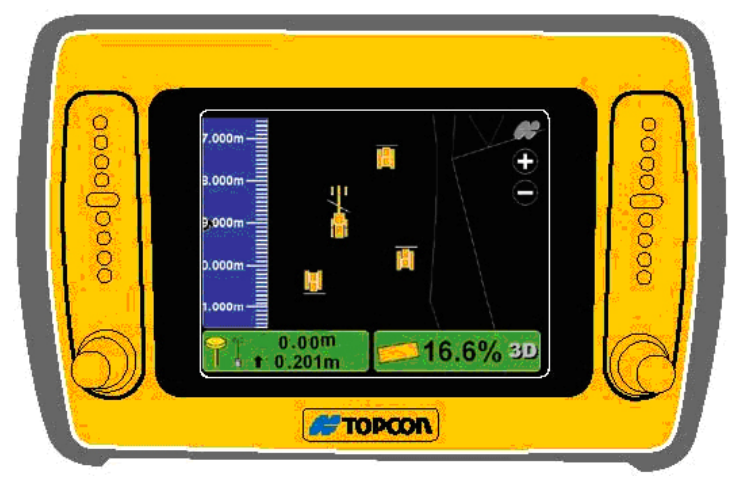

Figure 2: Graphical Display

\section{Automated systems using lasers}

The next big step forward in machine control uses fully automatics. Let's consider a bulldozer for this. It is one of the machines most often used in earthmoving. Actually, it is ideal for fully automatic control. As mentioned two laser receivers are used on the blade of the machine. Those receivers are to be placed so that they rise above the cab. This allows for almost unlimited operation, which is a big advantage when grading surfaces.

On site, the system works as follows: let's assume that the machine receiver does not pick up the laser beam in the middle of the reception area. Instead, it is received a little lower, say 3 $\mathrm{cm}$. This information is relayed to the control box, where all calculations take place. The control box commands the hydraulics to lower the blade. The blade is lowered and the process repeats. Now the laser receiver might see a deviation of $2.4 \mathrm{~cm}$. Again, the control box commands to lower the blade. This process is repeated until the receiver detects the ongrade position.

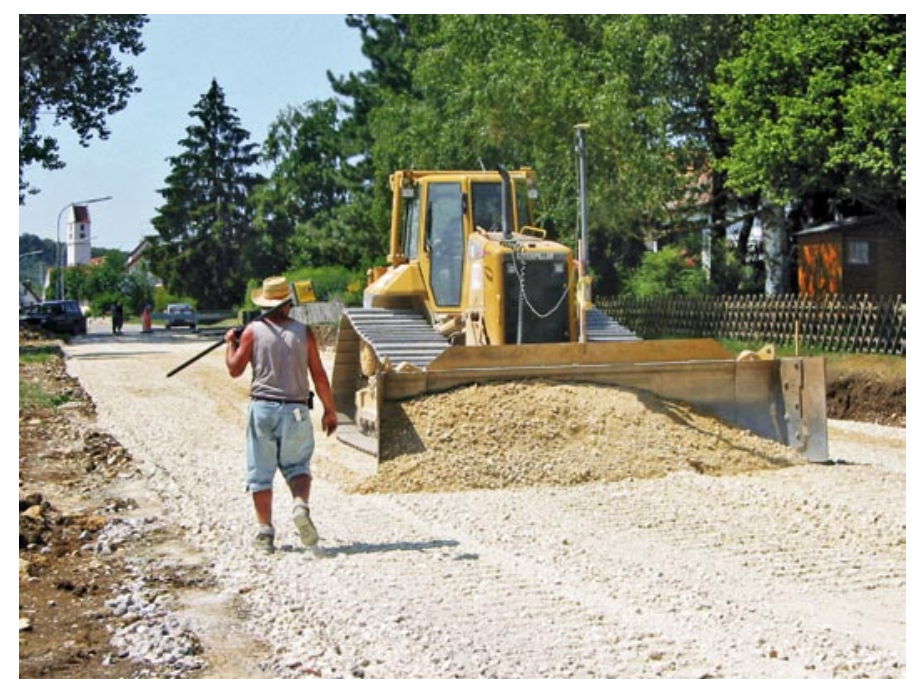

Figure 3: GPS automated dozer 
A normal rotating laser rotates with 600 Rotates Per Minute (RPM), meaning the receiver will receive approximately 10 signals per second. Even if the machine undulates due to the ground structure, this is enough to keep the blade on-grade. And that is the biggest advantage of an automated system: The surface is graded with less passes. Usually the design height is reached within two or three passes. To steer the hydraulics a valve block is connected parallel to the existing system. All the machine's standard features and functions are preserved. The machine can be used for any job, be it manually or automated, because the machine control system can easily be activated or deactivated. Some newer machines do no longer need a second hydraulic system to be installed and the machine control can be connected directly to the existing machine configuration, for example a joystick. The automated laser system described above is ideally suited for large areas like construction areas for halls, sports grounds or agricultural areas.

\section{Automated systems using sonic systems}

Road construction is another application that directly benefits from automated systems because large amounts of material are spread. The ideal solution for such long stretched sites is ultrasonic sensing. The sonic sensor is attached to one side and sweeps surfaces or measures the distance to a stringline. Sonic is not ideally suited for spreading material with a bulldozer, because the machine can not leave the reference (that is the stringline or the finished surface), which hinders the mobility a lot. For pavers or finishers, however, sonic solutions are ideal: They allow grading surfaces of arbitrary from with high accuracy.

Sonic, in contrast to laser, is an active sensor. This means the unit transmits and receives signals. The sonic sensor measures time, to work out distances. An ultrasonic pulse is transmitted and reflected on the reference plane, the stringline, etc. This reflected echo is what the sonic sensor receives. The time elapsed between transmitting and receiving is measured. As we know the speed of sound through air, this time can be used to calculate the distance. Temperature changes of the air (which occur when laying hot asphalt with the paver) are taken into account by measuring the temperature and applying a known reference distance.

There is a third group of sensors that do not use or need any active reference whatsoever. These are slope sensors used to determine the slope of blade and machine. That feature is of high interest when working with graders and dozers. The sensors "measure" slopes and can be set to design slopes received from the control box. Normally slope sensors are used in conjunction with other sensors. Very often, a combination of slope sensor and laser receiver or sonic system is used, depending on the application and type of machine.

\section{Three-dimensional machine control}

This technology uses all possible sensors to auto-control blade slope and grade for dozers or motor graders. GPS can be used with all mentioned sensors to control the machine; of course, total stations can be used as well.

GPS systems with one or two antennas on the blade are used to determine elevation and alignment of the machines blade. The design position of the blade is read from the 3D control box. The data has been entered into or transferred to the control box previously. This calculated design position is compared to the current position from the GPS. Corrections are fed into the automated system of the machine. Now the process resembles that of the laser control. The control box transfers a correction signal to the hydraulic system, which opens the relevant valves to move the blade. The process is repeated as often as needed to get design and current position to match. A three-dimensional machine control system enables to stakeout a digital design model with less work and higher accuracy. This is not only true for simply car parks, but for golf courses, highway exits and dumps or landfills or other complex terrains even more. 


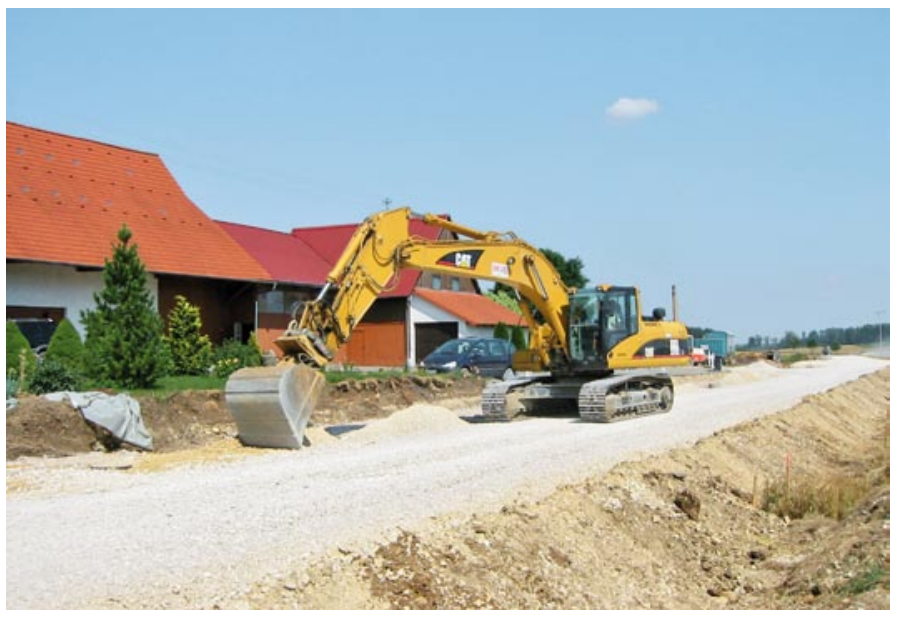

Figure 4: GPS indication on excavator

However, some requirements must be met for the system to be able to work properly: First, a digital terrain model (DTM) of the working area is needed. The system software allows importing many different file formats like REB, DWG, DXF, LandXML and ASCII. Creating the DTM directly in the software is possible, too.

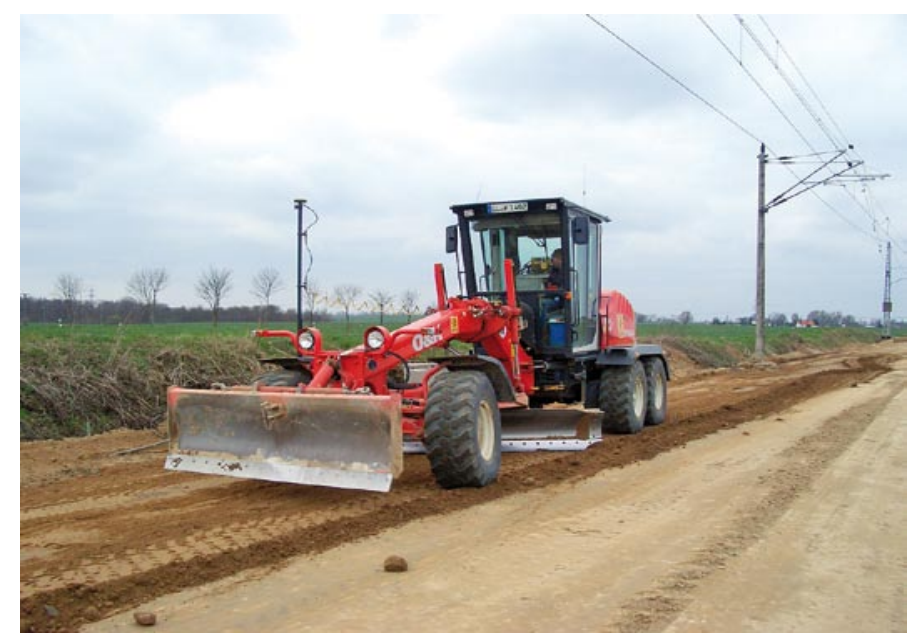

Figure 5: GPS automated Motor Grader

\section{GPS - how does it work?}

GPS is a system to determine positions using signals from satellites in orbit around the earth. The GPS positions need to be transformed into the coordinate system used on the construction site. To take care of this transformation a one-time calibration is needed for the site.

If the reference station is moved or another reference is used the calibration needs to be repeated. Using TOPCON software, you can measure a few points from the local system with the GPS to get their coordinates in the GPS system. After these control points have been measured, the transformation parameters are calculated automatically. Now the system can use the local coordinates. You no longer need to consider projection parameters or other calculations. 

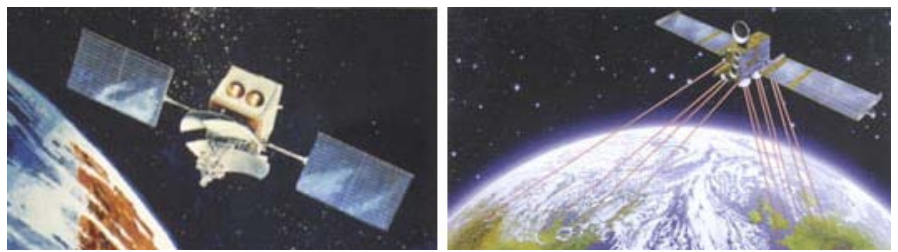

Figure 6: GPS and GLONASS satellites in orbit

\section{Site Automation - Does it pay off?}

Topcon offers comprehensive solutions to measure position and elevations for an optimized control of the complete production process on the construction site.

Topcon offers sophisticated and up-to-date solutions for any optical measuring devices, rotating lasers, machine control systems and GNSS survey products.

Topcon delivers modular solutions providing safety and efficiency. Without any interfacing hassle Topcon's automated machine control systems integrate survey and stake-out data, tailored to your requirements.

\section{Cost reduction:}

Reduction of machinery time (hours, fuel, blade wear)

Optimized use of materials by most accurate automatic control

Reduction of stake-out cost (material, work time)

\section{Increase in productivity:}

Up to four times faster execution of work

Uninterrupted and continuous production

Optimized use of materials - no remodeling

\section{Increase in quality:}

Permanently utmost accuracy

Constant quality during the whole process

Elimination of miscellaneous sources of error

\section{New technologies for machine control}

\section{SiteLink - a new communication and management system}

SiteLink is a communication system that combines performance data for machines and construction activities and reporting in one single application. It is a complete IT solution for global information management on construction site data.

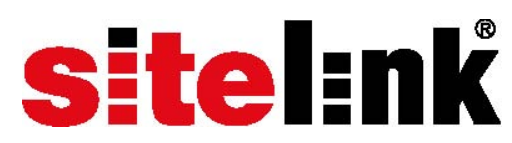

The SiteLink system supports different wireless communication options and processes data from the following areas: performance reports, data transfer, real time monitoring and remote support. One solution for all types and makes of construction equipment, regardless of manufacturer, age or technological issues. SiteLink is developed by TOPCON. 


\section{Communication system}

The new technology utilizes mesh radio and GPRS networks in a standard WiFi environment. The communication system tracks machines from all manufacturers on the site connected within the system.

The map display can be fine tuned via function keys: Zoom, navigation and several display options are available. SiteLink saves all information (all relevant machine data) permanently into a database that is available on a server. You can simply switch off the system at any time without having to save data or worry about data loss.

The following data can be collected, saved and edited per machine:

- Machine production

- Actual surface usage

- RTK status

- Actual surface off-set usage

- Productivity (according to different standards)

- Actual machine position in real-time utilizing an optional GPS receiver

- Percentage of finished projects

TOPCON's SiteLink software for tracking and reporting can be accessed via the internet. Thus it is a solution all around the world, be it a site office, your business head quarters or a machine on site.

\section{Planning and monitoring}

SiteLink is about to change the way contractors plan and monitor the operation of their construction equipment. Operators, site managers and others can get immediate updates on their moving assets - regardless of the location of those assets. Each machine connected to SiteLink can be found globally on any job site. Data can be transferred quickly and over large distances, too. A download to transfer data to a machine at a later point in time is possible as well.

\section{From machine to machine}

Another industry-unique feature is the possibility to link all operating machines to form a single mesh. This allows planners and operators to "talk" to each other. Individual linked machines take a double-function as repeaters, which provide information over a larger distance than the base telemetry itself would allow. This mesh for transmitting and receiving information optimizes the two-way communication between machines and the site center. Working like a LAN this new technology can optimize data exchange for the complete design and building process.

Operating mode and performance information for machines can be retrieved via mobile phone or internet. This data can be processed, forwarded or saved. No more vagueness and insecurities on site processes! You do not need to estimate a machines position, its current activity or the next maintenance schedule. You get all the data and every important piece of information in real-time. This allows for well-informed decisions.

\section{Always informed}

The site control centre communicates directly with any machine, for example to send recent job changes and new 3D model files to the machines and surveyors that need this data.

You save time and eliminate error sources as no physical transport of data or other job data is needed. Users of automated TOPCON maçhine control systems can take advantage of 
SiteLink real-time support.

Like some car-manufacturers can talk directly to their vehicles for driver support, SiteLink opens up a direct communication channel to the control system. This enables support in analyzing data and functions online and provide help to the operator.

\section{Paver and GPS - no accuracy problem!}

With the first 3D-mmGPS system for pavers TOPCON introduces the latest technology into the final stage of road construction. This real innovation is presented by Joseph Vögele AG: the new 3D control system NAVITRONIC ${ }^{\circledR}$ Plus.

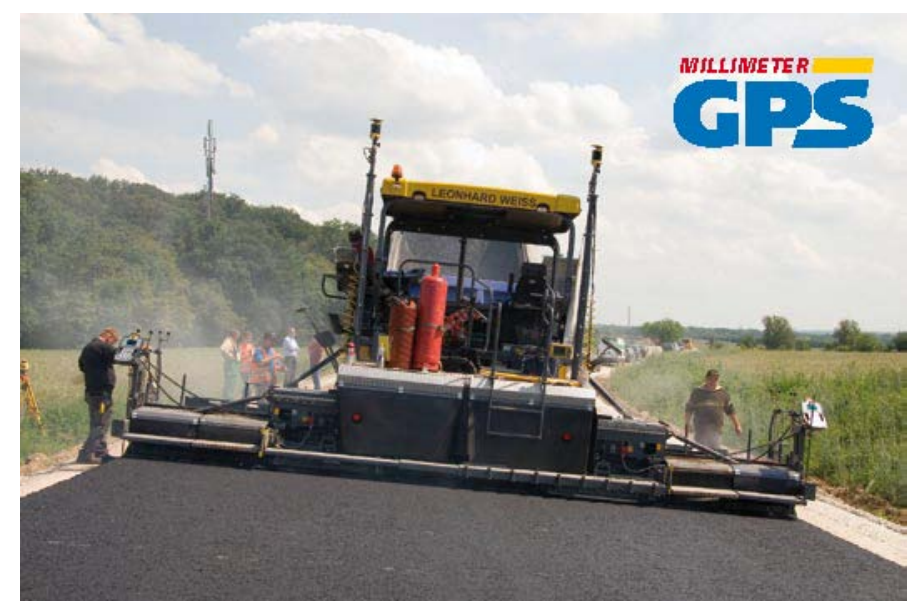

Figure 7: Vögele Paver with Topcon mmGPS

The total investment is drastically reduced by employing existing survey data (that has been used for earthworks) to control the paver.

Larger road construction projects, like highway construction or large squares, use 3D control for earthworks as a standard. For paving, however, the most common means are stringlines. Stringlines are used as a reference for elevation and position when paving. Stringing the stringlines is very time consuming and therefore needs a huge budget in each project. As NAVITRONIC ${ }^{\circledR}$ Plus uses a virtual reference the stringline work is gone. This way the system pays for itself in no time.

\section{Elevation and cross slope}

The central component of a 3D control system is the digital design model. It is connected to an open interface. Due to this interface the user has a choice of system, be it LPS with total station and prism or modern GPS or even mmGPS with zone laser. Millimeter GPS technology as used on pavers utilizes a zone laser, the PZL-1, which offers a working zone of $600 \mathrm{~m}$ (radius) by $10 \mathrm{~m}$ (height). A machine receiver like the PZS-MC is mounted to a pole installed on the pavers' screed. As soon as the sensor enters the working range of the laser zone signal the system supplies elevation corrections with millimeter accuracy.

\section{One correction - many machines}

Zone laser technology combines the rotating laser's simplicity, total station's precision and a GPS's flexibility to create an optimized, high-precision machine control system for motor graders and pavers. Of course, it can be used for dozers. GPS with millimeter accuracy can be used to stake-out or check heights behind the paver as well. By simply combining zone laser technology with any TOPCON GPS rover ang the PZL-1 receiver, you have high-precision 
elevation-check everywhere in your project.

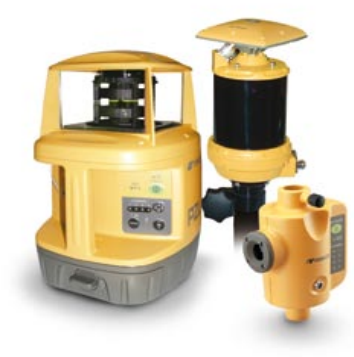

Figure 8: mmGPS components

Using similar positioning systems on machinery simplifies importing survey data, the socalled pavement design specification. Existing data can be fed to the machine for asphalt paving. This data usually is created during the sub base layers with graders and bulldozers. In conventional surveying, a new survey of the route has to be done after finishing the earthworks for building the superstructure. With NAVITRONIC ${ }^{\circledR}$ Plus this second survey is eliminated - the system re-uses the available data for the asphalt paving stage. In addition to saving time, there is another advantage: Surveyors and paving teams can work with the familiar system (total station or GPS) without instructions or (additional) training required.

\section{Automated control of direction}

The new 3D control system steers the paver directly. This allows full automatic control along the alignment using the current mmGPS position. The 3D control box display shows the paver's position as well as any corrections to reach the design model.
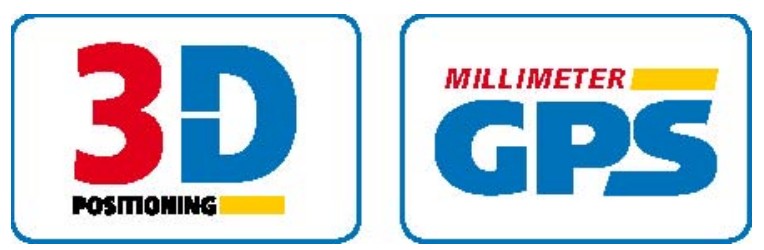

For the best paving accuracy, the screed's extending parts are precisely controlled. Sensors on the extending parts determine the screed's actual width. The user has two options to setup the horizontal precision: The paving reference is set to either the extreme left or the extreme right end of the screed. This line is then used as the paving reference. Optionally, users can set a fixed paving width. This will be adhered to over the full length of the section. Even direction corrections of the paver the reference alignment can be easily compensated for by using precise control of the screed's extending parts. Of course, the width control for the screed can be used when steering the paver manually. This ensures perfect paving.

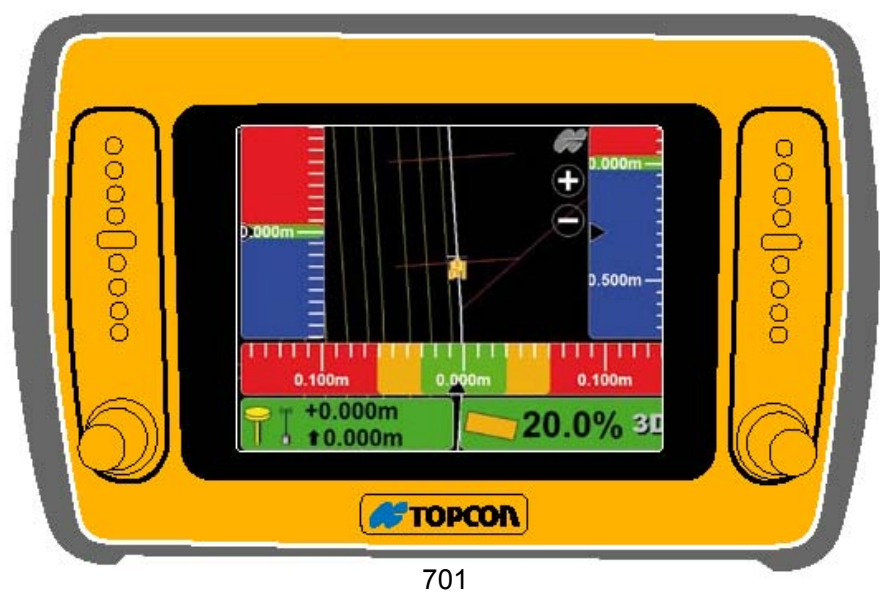




\section{Complete control of paving}

When using the system, the whole project is processed automatically. This is a crucial advantage if grade and slope are constantly changing. The system monitors the screed's grade, slope and position at all times and compares this data to the design data in the specification. Once the paver reaches a position where grade or slope need to be changed (for example in a bend), the screed is automatically adjusted to the new values.

Using a combination of all measured data (paver position, paving direction, screed position and slope) NAVITRONIC ${ }^{\circledR}$ Plus is capable of strictly controlling the paving process at any point of the planned route.

Combining mmGPS and NAVITRONIC ${ }^{\circledR}$ Plus is your warranty for paving: no accuracy and productivity problems. 